\title{
An efficient local clustering approach for simplification of 3D point-based computer graphics models
}

\author{
Zhiwen $\mathrm{Yu}$ \\ Department of Computer Science \\ City University of Hong Kong \\ yuzhiwen@cs.cityu.edu.hk
}

\author{
Hau-san Wong \\ Department of Computer Science \\ City University of Hong Kong \\ cshswong@cityu.edu.hk
}

\begin{abstract}
Given a point-based $3 D$ computer graphics model which is defined by a point set $P\left(P=\left\{p_{i} \in R^{3}\right\}\right)$ and a desired reduced number of output samples $N_{s}$, the simplification approach finds a point set $P_{s}$ which (i) satisfies $\left|P_{s}\right|=N_{s}\left(\left|P_{s}\right|\right.$ is the cardinality of $\left.P_{s}\right)$ and (ii) minimizes the difference of the corresponding surface $S_{s}$ (defined by $P_{s}$ ) and the original surface $S$ (defined by $P$ ). Although a number of previous approaches have been proposed for simplification, most of them (i) do not focus on pointbased 3D models, (ii)do not consider efficiency, quality and generality together. In this paper, we introduce an adaptive simplification method (ASM) which is an efficient technique for simplifying point-based complex $3 D$ model. ASM achieves low running time by clustering the points locally based on the preservation of geometric characteristics. Finally, we analyze the performance of ASM and show that it outperforms most of the current state-of-theart methods in terms of efficiency, quality and generality.
\end{abstract}

\section{Introduction}

Early work in the simplification of 3D computer graphics model focuses on polygonal models because other model representations, such as spline, volumetric and implicit-surface can be converted to a polygonal one. There are four main types of algorithms for simplifying polygonal meshes: (i) Vertex removal (Figure 1(a)) (e.g. [1]); (ii) Vertex clustering (Figure 1(b)) (e.g. [2], [3]); (iii) Edge contraction(Figure 1(c)) (e.g. [4], [5]); (iv) Particle simulation(Figure 1(d))(e.g. [6], [7]). However, most of the above algorithms cannot be applied to point-based surface directly. The main motivation of this paper is to investigate whether there is a new approach which $(i)$ inherits the advantage of simplification algorithms for polygonal meshes and $(i i)$ works well on point-based surface.

With the popularity of 3D scanners, more and more point-based 3D models are available. Point-based 3D models have three characteristics: $(i)$ most of them consists of millions of 3D points; (ii) the boundary surfaces of the models are represented by a discrete point set; (iii) there does not exist any topological relationship between these points. Traditional methods first convert a discrete point cloud to a continuous surface representation, such as spline, volumetric, implicit-surface, or polygon. Then, the mod-

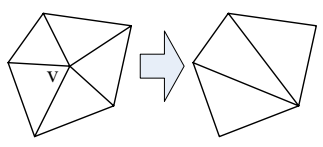

(a) Vertex removal

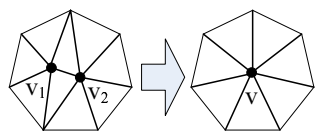

(c) Contraction

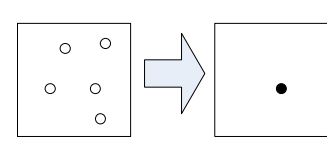

(b) Vertex clustering

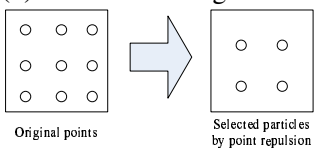

(d) Particle simulation
Figure 1. Different kinds of simplification methods

els are simplified by the above simplification algorithms. However, the process of conversion is time consuming and memory consuming, especially with the increasing cardinality of the point set. As a result, another motivation of this paper is to investigate whether there is a new approach which can simplify the point set directly and avoid the process of conversion.

The contribution of this paper are threefold. First, we propose a local clustering approach which is very efficient for clustering the points in a grid cell structure based on the preservation of geometric characteristics. Second, we introduce a novel error metric, known as key point error (KPE), to evaluate the error of the simplified model. Third, we introduce an adaptive simplification method (ASM) which combines local clustering with a global error-bound.

The rest of the paper is organized as follows. Section 2 surveys the related work on simplification methods for point-based models. Section 3 describes local clustering methods, key point error, and the ASM algorithm. Section 4 evaluates ASM experimentally. Section 5 is conclusion and future work.

\section{Background}

Recently, point clouds receive much attention as a representation of models in computer graphics. Point clouds which are produced by 3D scanning devices can be rendered and visualized directly by point-based rendering and visualization methods.

Alexa et al.[8] and Linsen et al[9] first proposed simplification algorithm for point-based models. Their algorithms belong to the class of vertex removal. Then, Pauly and Gross[10] provided a resampling strategy based on one of signal processing theory. The most related work to us is proposed by Pauly, et al [11]. They 
provided four surface simplification methods which are adapted from the simplification methods for polygonal surface: incremental clustering, hierarchical clustering, iterative simplification and particle simulation.

\section{Adaptive simplifi cation algorithm}

\subsection{Key point}

Without loss of generality, we assume that a point-based surface $S_{c}$ in a grid cell $c$ can be approximated by a local surface function $f(p)$. Usually, local surface function is a polynomial function which is determined by a set of points $p_{i}\left(p_{i} \in c, 1 \leq i \leq n_{c}\right.$, where $n_{c}$ is the number of the points in a grid cell $c$ ). The point $p_{j}$ ( $p_{j} \in c, 1 \leq j \leq n_{k} \leq n_{c}$, where $n_{k}$ is the number of the key points in $c$ ) is called a "Key point" if its contribution is high in the process of determining the geometric characteristics of a local surface. Figure 2 illustrates the key points(the black points) on the curve in a 2D view. Key points in figure 2(a) determine the extent of the curve and the maximum value and the minimum value of the curve. If key points are preserved, most of the geometric characteristics of the curve are preserved as well in the process of simplification. Key points include three kinds of points: $(i)$ start points and end points; (ii) stationary points; (iii) inflection points. These key points capture the skeleton of the curve or the surface. The preservation of key points decrease the difference between the original surface $S_{c}$ before simplification and the corresponding surface $S_{c}^{\prime}$ after simplification.

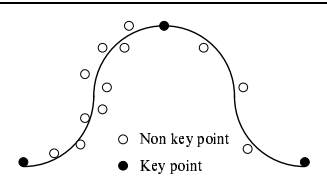

(a) key points of the curve

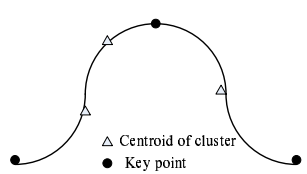

(b) simplifying the curve
Figure 2. Key point cording to their corresponding eigenvalues.Note that, the objective here is to determine the variance of the local surface normal. Finally, all the points are projected to the new coordinate system which is formed by the eigenvectors. The following Lemma 1 and Lemma 2 are obtained by the property of key points.(We only focus on stationary points)

Lemma 1. If the coordinate of a point $p$ along the axis $v_{2}$ with the smallest eigenvalue has the maximum (minimum)value, $p$ is a global maximum(minimum) point in grid cell $c$.

Lemma 2. If the coordinate of a point $p$ along the axis $v_{2}$ with the smallest eigenvalue is greater(smaller) than that of its neighbors, $p$ is a local maximum (minimum)point in grid cell $c$.

The proofs are given in [13].

There are two ways for the algorithm to determine the key points according to the lemmas:(i) It obtains the points with the maximum or minimum value along the axis $v_{2}$ as key points directly; (ii) It considers the points one by one. If the point satisfies Lemma2, the point is a local maximum (minimum) point. Although searching for the nearest neighbor of a point is very fast, computational cost will be expensive for a large point set. In order to save computation cost, the algorithm adopts a partition strategy: $(i)$ it calculates the difference between the maximum value $M A X_{v_{2}}$ and the minimum value $M I N_{v_{2}}$ along $v_{2}$. (ii) A partition threshold $\alpha\left(0 \leq \alpha \leq \frac{1}{2}\right)$ is specified. There exists two planes perpendicular to $v_{2}$ and whose intersections with $v_{2}$ are given by $M A X_{v_{2}}-\alpha \cdot\left(M A X_{v_{2}}-M I N_{v_{2}}\right)$ and $M I N_{v_{2}}-\alpha \cdot\left(M A X_{v_{2}}-M I N_{v_{2}}\right)$ respectively. They divide the space in the grid cell $c$ into three parts. (iii) The algorithm clusters the points in the three parts based on another two axis $v_{0}, v_{1}$. Note that, we take the center of the clusters above the plane $v_{2}=\left(M A X_{v_{2}}-\alpha \cdot\left(M A X_{v_{2}}-M I N_{v_{2}}\right)\right)$ or below the plane $v_{2}=M I N_{v_{2}}-\alpha \cdot\left(M A X_{v_{2}}-M I N_{v_{2}}\right)$ as key points, since adopting the center of the clusters $(i)$ alleviates the influence of noise and (ii) the centers are close to the key points if $\alpha$ is small. The value of $\alpha$ determines the number of missing key points. If $\alpha$ is large, the number of missing key points is small. However, in this case, the center of the clusters are not close to the key points any more and cannot achieve the objective of preserving key points. According to our experiments, $\alpha=\frac{1}{8}$ represents a suitable choice.

The algorithm is divided into six steps: $(i)$ the mean point $p_{c}$ of all the data points $p_{i}$ in the grid cell $c$ is computed by the equation $p_{c}=\frac{\sum_{i=1}^{n_{c}} p_{i}}{n_{c}}\left(n_{c}\right.$ is the number of points in a grid cell $c$ ). (ii) A $3 \times 3$ covariance matrix $C_{M}$ is calculated by the equation $C_{M}=\operatorname{Cov}($ axis $k$, axis $l)=\frac{\sum_{i=1}^{n_{c}}\left(p_{k i}-p_{k c}\right)\left(p_{l i}-p_{l c}\right)}{n_{c}}$, where axis $k$, axis $l \in\{x-$ axis, $y-$ axis, $z-$ axis $\}$. (iii) the eigenvectors and eigenvalues of the covariance matrix $C_{M}$ are determined by the equation $C_{M} \cdot v_{k}=\lambda_{k} \cdot v_{k}(0 \leq k \leq 2)$. (iv) All the points are transformed to the new coordinate system defined by the eigenvectors $v_{0}, v_{1}, v_{2}$. $(v)$ The points are partitioned into three parts by the above partition strategy. (vi)The points in the top and bottom parts are clustered as key points, while the points between the key points are clustered by their values along $v_{0}, v_{1}$. The centroids of the clusters become the new sample points.

Figure 3 illustrates this local clustering approach based on PCA using a 2D view. Figure 3(a) shows the original data points, 


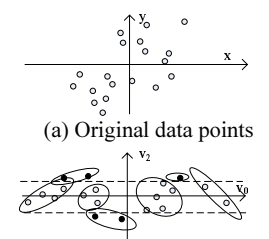

(c) Partition strategy

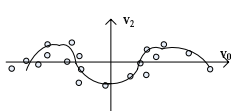

(b) PCA transform

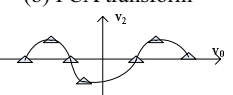

(d) New samples
Figure 3. Local clustering

while Figure 3(b) illustrates the change after PCA. The points in Figure 3(c) are partitioned into three parts by two dashed lines $\left(v_{2}=M A X_{v_{2}}-\alpha \cdot\left(M A X_{v_{2}}-M I N_{v_{2}}\right)\right.$ and $v_{2}=M I N_{v_{2}}-\alpha$. $\left.\left(M A X_{v_{2}}-M I N_{v_{2}}\right)\right)$ according to the partition strategy. It is obvious that there are two clusters in the top partition, while only one cluster is in the bottom. The centers of the clusters which contain the black points are viewed as key points. Finally, all the points in the middle part are clustered between the key points along $v_{0}$. Figure 3(d) shows the new samples after clustering.

Figure 4 shows the results of the local clustering method based on PCA to simplify the model of a dragon (437646 points) to different output model size $\left(N_{s}\right)$. The arrows in the top row show some key points which are preserved by the algorithm, while the arrows in the bottom row point out the change in mean curvature.

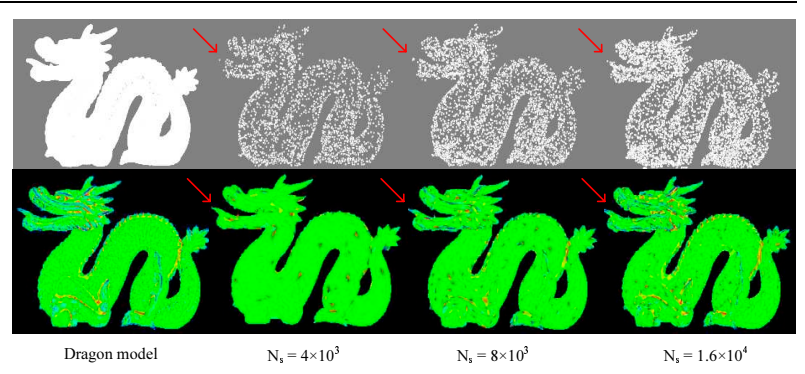

Figure 4. Dragon model

\subsection{Combination with random sampling}

The clustering algorithm has two major disadvantages: $(i)$ the surface quality is low; $(i i)$ we are unable to control the number of samples. The objective of key point preservation is to improve the quality of the surface. In order to control the number of the output samples, we combine local clustering with random sampling. First, the system gives the number of samples $N_{c}$ for the grid cell $c$. Then, the algorithm clusters the black points in Figure 3(c).(iii) if $\left(N_{c}>C_{n}\right)$ (where $C_{n}$ is the number of the centers of the clusters), it selects $N_{c}-C_{n}$ samples by clustering the white points. (iv) if $\left(N_{c}<C_{n}\right)$, it randomly selects $N_{c}$ from $C_{n}$ points.

\subsection{Error analysis}

We propose a novel error estimation method based on the observation that the output surface $S^{\prime}$ is similar to the original surface $S$ which means the key points in $S^{\prime}$ are close to the corresponding key points in $S$. The error between $S^{\prime}$ and $S$ is measured by the average of the distances between key points, which we refer to as the Average key point error(AKPE). We can also adopt a Maximum key point error(MKPE) as defined in $\mathrm{Eq}(2)$ below:

$$
\begin{array}{r}
A K P E=\frac{1}{n_{k}} \sum_{k=1}^{n_{k}} d\left(p_{k}[i], p_{k}^{\prime}[i]\right) \\
M K P E=\operatorname{Max}_{1 \leq k \leq n_{k}} d\left(p_{k}[i], p_{k}^{\prime}[i]\right) \\
d\left(p_{k}[i], p_{k}^{\prime}[i]\right)=\sum_{i=0}^{2}\left(p_{k}[i]-p_{k}^{\prime}[i]\right)^{2}
\end{array}
$$

where $n_{k}$ is the number of selected key points, $p_{k}[i]$ is the coordinate of the key $\operatorname{point}\left(p_{k} \in S\right)$ along the $i$ th dimension, $p_{k}^{\prime}[i]$ is the coordinate of the corresponding key point $\left(p_{k}^{\prime} \in S^{\prime}\right)$. $d\left(p_{k}[i], p_{k}^{\prime}[i]\right)$ is the distance measure.

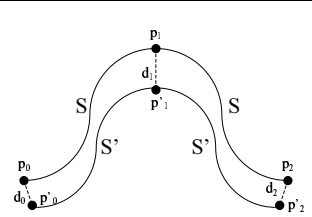

(a) error estimation

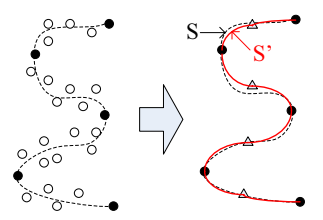

(b) local cluster
Figure 5. Error estimation

Figure 5(a) illustrates the process of calculating KPE. The algorithm first obtains the key points $\left\{p_{0}, p_{1}, p_{2}\right\}$ in the original surface $S$ and the corresponding key points $\left\{p_{0}^{\prime}, p_{1}^{\prime}, p_{2}^{\prime}\right\}$ in the output surface $S^{\prime}$ by the methods in section 3.2. Then, the distances $\left(d_{0}, d_{1}, d_{2}\right)$ between key point pairs $\left(p_{0}, p_{0}^{\prime}\right),\left(p_{1}, p_{1}^{\prime}\right),\left(p_{2}, p_{2}^{\prime}\right)$ are computed respectively. Finally, KPE is calculated by Eqs (1) and (2).

\subsection{The overview of the algorithm}

The algorithm for ASM is shown in Figure 6. In order to control the total number of output samples, an evaluation function $f\left(c_{i}\right)$ is proposed to determine the contribution of each grid cell $c_{i}$ as follows.

$$
f\left(c_{i}\right)=\frac{\omega \cdot c_{s} \cdot\left|c_{i}\right|}{\left|P_{s}\right|}
$$

where $\omega$ is the value of weight which reflects the importance of the grid cell $c_{i}, c_{s}$ is the output model size, $\left|c_{i}\right|$ is the number of the points in the grid cell $c_{i}$, and $\left|P_{s}\right|$ is the cardinality of the point set $P_{s}$. Obviously, the value of $f\left(c_{i}\right)$ is proportional to $\left|c_{i}\right|$.

The error tolerance $\epsilon$ is given by the user which bounds the maximum key point error (MKPE)for local clustering. If MKPE is greater than $\epsilon$ in the process of local clustering, we reduce the value of $\alpha(\alpha$ is the control parameter to determine the partition plane) and adjust the corresponding partition plane by the fitness function $\psi(\alpha)$

$$
\psi(\alpha)=\frac{M A X_{v_{2}}-\frac{\left(M A X_{v_{2}}-\alpha_{1} \cdot\left(M A X_{v_{2}}-M I N_{v_{2}}\right)\right) \cdot \epsilon}{M K P E}}{M A X_{v_{2}}-M I N_{v_{2}}}
$$

where $M A X_{v_{2}}$ and $M I N_{v_{2}}$ are the maximum and minimum value along the eigenvector $v_{2}$ with the smallest eigenvalue respectively, and $\alpha_{1}$ is the value of $\alpha$ before adjusting. Maximum 
key point error $M K P E$ is then computed by Eq (2). Note that, it is obvious that the key point with maximum key point error is the global maximum point or the global minimum point in the grid cell $C_{i}$. As a result, the algorithm first calculates $M K P E$. If $M K P E>\epsilon$, it adjusts the value of $\alpha$ by Eq (5). Otherwise, it clusters other points by the approach based on PCA.

Algorithm ASM(Point set $P$, Sample number $N_{s}$, Tolerance $\epsilon$ )

$I^{*} \epsilon$ is the maximum error tolerance $* /$

$I^{*}\left|P_{s}^{\prime}\right|$ is the cardinality of the point set $P$ after clustering $* /$

I*MKPE$E_{N}$ is the key point error after clustering */

1. Hashes all the points to the grid $\mathrm{G}$

2. For each grid cell $c_{i}$

Compute $f\left(c_{i}\right)$ by $\mathrm{Eq}(4)$;

Calculate $M K P E$ by $\mathrm{Eq}(2)$;

If $(M K P E>\epsilon)$

Compute $\alpha$ by Eq(5);

Perform local clustering;

\section{Figure 6. The algorithm for ASM}

\section{Experiment}

All the experiments presented are executed with a Pentium $2.8 \mathrm{GHz}$ CPU with 1 GByte memory. Our datasets are obtained from the Stanford 3D scanning repository([12]), and other geometric models archives. The cardinality of the models are shown in Table 1 . The time for simplifying models (the size of output model is $5 \%$ of the size of input model) and the Average key point errors(AKPE) are illustrated in Table 2

\begin{tabular}{|llllll|}
\hline Model name & \multicolumn{2}{l}{ Cardinality Output } & Model name & Cardinality Output \\
\hline Human Brain & 19459 & 973 & Knee & 37889 & 1895 \\
Ball Joint & 137063 & 6854 & Armadillo & 172975 & 8649 \\
Skeleton hand & 327324 & 16366 & Dragon & 437646 & 21882 \\
Hip & 530169 & 26509 & HappyBuddha & 543653 & 27183 \\
Malaysia & 1815771 & 90789 & Asian Dragon & 3609601 & 180481 \\
Thai Statue & 4999997 & 250000 & Lucy & 14027873 & 701394 \\
\hline
\end{tabular}

Table 1. The cardinality of the models

\begin{tabular}{|llllll|}
\hline Model name & \multicolumn{2}{l}{ time(s)AKPE } & Model name & time(s) AKPE \\
\hline Human Brain & 0.126 & $7.03 \times 10^{-5}$ & Knee & 0.211 & $6.83 \times 10^{-5}$ \\
Ball Joint & 0.843 & $6.52 \times 10^{-5}$ & Armadillo & 1.421 & $6.51 \times 10^{-5}$ \\
Skeleton hand & 2.112 & $6.48 \times 10^{-5}$ & Dragon & 2.314 & $6.47 \times 10^{-5}$ \\
Hip & 3.161 & $6.41 \times 10^{-5}$ & Happy Buddha & 3.161 & $6.41 \times 10^{-5}$ \\
Malaysia & 8.121 & $6.27 \times 10^{-5}$ & Asian Dragon & 15.061 & $6.13 \times 10^{-5}$ \\
Thai Statue & 25.014 & $6.01 \times 10^{-5}$ & Lucy & 35.263 & $5.81 \times 10^{-5}$ \\
\hline
\end{tabular}

Table 2. The simplification time and AKPE

Figure 7 illustrates the results of ASM when applied to simplify a ball joint model (137063 points) to different output model size $\left(N_{s}\right)$. The arrows in the top row show some key points which are preserved by the algorithm, while the arrows in the bottom row point out the change in mean curvature. Blue color denotes high curvature, while green color denotes low curvature. Note that, the key point preservation leads to the changes in mean curvature around the key point. In general, the change of the mean curvature is determined by the distribution of the points around the key point.

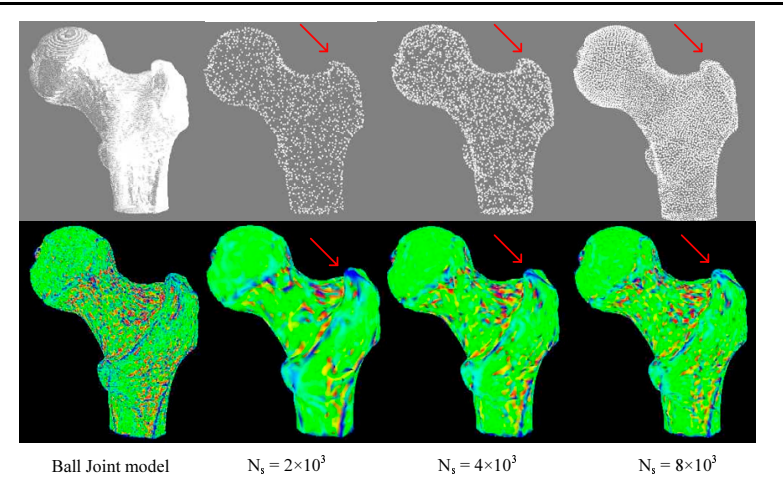

Figure 7. Ball joint model

\section{Conclusion and future work}

The paper investigates the problem of meshless simplifi cation. Our contribution is an effi cient simplifi cation method which we refer to as the adaptive simplifi cation method(ASM). ASM is based on PCA which preserves most of the key points with a small computational cost. A key point error metric is then introduced to measure the difference between the original surface and the surface after simplifi cation. ASM guarantees a global error bound in the simplifi cation process. In the future, it will be interesting to apply ASM to different kinds of point-based models with different surface properties.

\section{Acknowledgments}

The work described in this paper was partially supported by grants from the Research Grants Council of Hong Kong Special Administrative Region, China [Project No. CityU 1197/03E and CityU 121005] and grants from City University of Hong Kong [Project No. 7001596 and 9360091].

\section{References}

[1] William J. Schroeder, Jonathan A. Zarge, and William E. Lorensen. Decimation of triangle meshes. Computer Graphics (SIGGRAPH 92 Proc.), 26(2):6570, July 1992

[2] Jarek Rossignac and Paul Borrel. Multi-resolution 3D approximations for rendering complex scenes. In B. Falcidieno and T. Kunii, editors, Modeling in Computer Graphics: Methods and Applications, pages 455-465, 1993.

[3] David Luebke and Carl Erikson. View-dependent simplifi cation of arbitrary polygonal environments. In SIGGRAPH 97 Proc., August 1997.

[4] Garland, M., Heckbert, P. Surface simplifi cation using quadric error metrics. SIGGRAPH 97, 1997.

[5] Michael Garland, Yuan Zhou. Quadric-based simplifi cation in any dimension . ACM Transactions on Graphics (TOG), Volume 24 Issue 2.April 2005.

[6] Turk, G. Re-Tiling Polygonal Surfaces. SIGGRAPH 92,1992.

[7] Witkin, A., Heckbert, P. Using Particles To Sample and Control Implicit Surfaces. SIGGRAPH 94, 1994.

[8] Alexa,M., Behr, J., Cohen-Or, D., Fleishman, S., Levin, D., Silva, T. Point Set Surfaces, IEEE Visualization 01, 2001.

[9] Linsen, L. Point Cloud Representation. Technical Report, Faculty of Computer Science, University of Karlsruhe, 2001.

[10] Pauly, M., Gross, M. Spectral Processing of Point-Sampled Geometry, SIGGRAPH 01, 2001.

[11] Mark Pauly, Markus Gross, Leif P. Kobbelt. Effi cient Simplifi cation of PointSampled Surfaces. Proceedings of the conference on Visualization '02.October 2002 .

[12] http://www-graphics.stanford.edu/data/3Dscanrep/

[13] ZhiWen Yu, Hau-San Wong, "An effi cient local clustering approach for simplifi cation of 3D point-based computer graphics models", Technical report, 2006. 